\title{
The efficacy of a virtual reality exposure therapy treatment for fear of flying: A
}

\section{retrospective study}

Amihai Gottlieb ${ }^{1}$, Glen M. Doniger ${ }^{1,2,3}$, Yara Hussein ${ }^{1}$, Shlomo Noy ${ }^{4}$, Meir Plotnik**1,5

1 Center of Advanced Technologies in Rehabilitation, Sheba Medical Center, Ramat

Gan, Israel

2 The Joseph Sagol Neuroscience Center, Sheba Medical Center, Ramat Gan, Israel

3 Baruch Ivcher School of Psychology, Interdisciplinary Center (IDC) Herzliya,

Herzliya, Israel

4 Department of Psychiatry. Sheba Medical Center, Ramat Gan, Israel

5 Faculty of Medicine \& The Sagol School of Neuroscience, Tel Aviv University, Tel

Aviv, Israel

* corresponding Author: Dr. Meir Plotnik

Meir.potnik@sheba.health.gov.il

Sheba Medical Center, Ramat Gan 52621, Israel. 
Efficacy of a VRET for FOF

\section{Abstract}

Background: Fear of flying (FoF) is an anxiety disorder classified as a phobia. Its prevalence is estimated at $10-40 \%$ in the industrialized world, and it is accompanied by severe economic, social, vocational and emotional consequences. In recent years, virtual reality-based exposure therapy (VRET) for FoF has been introduced. One such FoFVRET is offered as a paid clinical service at the Center of Advanced Technologies in Rehabilitation (CATR), Sheba Medical Center, Israel. Positive long-term efficacy of FoF-VRET has been found in several studies. However, these studies are limited by relatively small, non-representative samples and a lack of comparative pre/post functional efficacy outcome measures. Methods: To address these limitations, we conducted a retrospective survey of self-referred individuals treated with FoF-VRET at CATR over the previous four years. The aim of the present study was to evaluate the efficacy of our FoF-VRET in this representative real-world sample. Of 274 individuals who received the treatment, 214 met inclusion/criteria, and 103 agreed to participate. The survey focused mainly on collecting information regarding flight activity before and after treatment. The primary outcome measures were: (1) number of flights per month (FpM); (2) number of flight hours per month (FHpM). For each participant, these outcomes were computed for the post-treatment period ( $\geq 6$ months after FoF-VRET) and the corresponding pretreatment period. Results: FpM (mean \pm SD) increased from $.05 \pm .07$ to $.16 \pm .07$ flights $(p<.0001)$. FHpM rose from.22 \pm .41 to $.80 \pm .86$ hours per month $(p<.0001)$. Conclusions: These results are indicative of FoF-VRET treatment efficacy. Future studies should evaluate long-term maintenance of the treatment effect and thus identify the optimal frequency for delivery of periodic booster treatments. 
Efficacy of a VRET for FOF

Keywords: Anxiety Disorders; Treatment; Empirical supported treatments; Computer/Internet Technology; Behavior Therapy; Phobia/Phobic Disorders

\section{INTRODUCTION}

Airplanes are the safest, most common way to travel in the industrialized world $(1,2)$. Fear of flying (FoF) is a common anxiety disorder in western countries, and its prevalence is estimated at 10-40\% (3-5). Among those who suffer from FoF, $14 \%$ have never flown on an airplane, $6 \%$ have flown and say they will not fly again, and $10 \%$ have flown and say they will fly again only if there is no other choice (6). FoF may be secondary to phobias related to environmental conditions (e.g., altitude, severe weather) or situational phobias (e.g., claustrophobia), and may be comorbid to panic attacks and generalized anxiety disorder (7). Physiological and psychological anxiety symptoms of FoF may include panic attacks, fear, muscle tension, sweating, shortness of breath, heart palpitations, nausea and dizziness (8). The costs of FoF for affected individuals, their families and society are substantial. FOF sufferers tend to avoid flying entirely, which may have serious social, vocational and emotional consequences (9). Societally, FoF results in significant cost to the airlines and incalculably reduced productivity and opportunity (9).

Several pharmacological treatments exist for FoF including anti-anxiety medications like benzodiazepines (10). Other treatments are psychological interventions like exposure therapy (also called systematic desensitization) (11). The most common treatment for FoF is cognitive behavioral therapy (CBT) (12), which focuses on creating neutral memories to replace the panic-inducing ones, and may include relaxation techniques, psychoeducation and exposure therapy. In exposure therapy, the FoF sufferer is exposed 
Efficacy of a VRET for FOF

to the source of anxiety in a controlled manner, and this approach is considered the most efficient treatment for FoF $(13,14)$. Exposure therapy for FoF might involve simulating a flight or exposure to a stationary plane.

Over the last decade, virtual reality (VR) has become a viable method for administering exposure therapy for anxiety disorders. For example, several VR-based exposure treatments for post-traumatic stress disorder have been proposed [for review see (15)]. As applied to FoF, virtual reality exposure therapy (VRET) involves creating a virtual airplane environment that simulates various aspects of flying using dynamic visual, auditory and motion stimuli (7). Unlike exposure therapy using a real flight, this VR-based method allows the therapist to systematically control the level of the exposure intensity to a variety of elements (16). Notably, VRET for FoF (FoF-VRET) is most often implemented with a VR head mount display (e.g., $(17,18))$ and thus lacks the ability to simulate motion. Large-scale VR systems that incorporate motion can be used to address this limitation and better simulate the flight experience.

There are few reports evaluating the clinical efficacy of FoF-VRET [e.g. (13, 17-22)]. In a recent meta-analysis of 11 randomized trials, Cardos and colleagues found FoFVRET to be superior to control/standard FoF treatments (23). Only a few randomized trials have assessed efficacy in the months following treatment. For example, Rothbaum and colleagues (2000) (21) reported maintenance or enhancement of self-reported posttreatment improvements after six months for both VRET and standard exposure therapy groups. Further, at six months post-treatment, $79 \%$ of VRET participants and $69 \%$ of standard exposure therapy participants reported that they had flown (voluntarily) since completing the treatment. In another study, Muhlberger and colleagues (2003) (17) found 
Efficacy of a VRET for FOF

71 that $62 \%$ of VRET participants reported flying during the 6-month follow-up period.

72 However, Maltby and colleagues (2002) (20) reported that differences between VRET

73 and an attention-placebo group observed immediately following treatment had

74 disappeared after six months. In a randomized controlled trial, Rothbaum and colleagues

75 (2002) (24) found that 92\% of VRET and 91\% of standard exposure participants had

76 flown one-year post-treatment. Tortella-Felui (2011) (18) found that 66\% of VRET

77 participants reported flying during the 1-year follow-up period. Finally, in a long-term

78 follow-up study, Wiederhold (2003) (25) found that $85 \%$ of their 30 participants reported

79 flying in the three years after completing several different VRET treatments. that there is great variability $(62-92 \%)$ in the prevalence of (voluntary) flying in the period following the conclusion of VRET treatment $(17,24)$. Further, participants in such studies are not considered representative of the general population as they have consented to an experimental treatment and are thus particularly motivated and amenable to the treatment. Most importantly, existing studies lack comparative pre/post functional

86 efficacy outcome measures. To address these issues, better controlled studies with larger, more representative clinical samples are needed.

90 See Czerniak and colleagues (2016) (7) for a full description of the setup (see also

91 Methods). To date (January 2019) more than 274 individuals have been treated.

The aim of the present study was to evaluate the efficacy of our FoF-VRET by

93 retrospectively surveying individuals who received the treatment as a paid clinical 
Efficacy of a VRET for FOF

94 service. Our primary objective was to evaluate whether flying habits changed after 95 completion of the treatment.

Rationale

98 Between 2014 and 2018, 274 individuals were self-referred to receive FoF-VRET at

99 CATR. We emailed 214 individuals who had completed the treatment and for whom we

100 had an email address on file. In the email, we asked if they would be willing to 101 participate in a phone survey regarding the FoF treatment they received (see Procedure 102 for more details). Among the benefits of this methodology are: (1) reduced bias 103 associated with willingness to participate in experimental research; (2) reduced bias 104 associated with an onsite office interview by a clinician; (3) reduced 'gratitude effect' 105 consequent to pro bono research participation; participants in the present study paid out106 of-pocket to obtain a clinical service.

107 Participants

108 Inclusion criteria - (1) completion of the FoF-VRET treatment regimen at CATR; (2) 109 email address on file (to facilitate emailing of consent at initial contact).

110 Exclusion criteria - (1) non-responsive to email; (2) refusal to participate; (3) <6 111 months after treatment completion.

112 Six months was set as the minimum time from treatment completion to allow a 113 reasonable amount of time for participants to fly and for comparability to the literature 114 (see Main outcome measures). Of the 214 individuals we contacted, 103 actually 115 participated. Individuals were excluded for the following reasons: 50 were non- 
Efficacy of a VRET for FOF

116 responsive, 53 refused to participate and 8 were questioned $<6$ months from treatment

117 completion.

118

119

120

121

122

123

124

125

\section{Procedure}

First, potential participants were emailed for their consent to participate; those who agreed were then contacted by phone to confirm their informed consent. Next, a structured phone interview was conducted. The interview consisted of three parts:

(1) Confirmation of FoF-VRET treatment dates and recording the reason or reasons for self-referral.

(2) Information on flight activity for the period following treatment completion and a corresponding period of identical length of time prior to treatment initiation. For each flight, participants reported their destination and flight duration. In addition, participants rated their anxiety level during each flight on a scale from 1 (least anxious) to 7 (most anxious). Only outbound flights were recorded (i.e., flights departing Ben Gurion Airport, Israel). For verification purposes, participants were asked to furnish supporting material, including boarding passes and passport stamps.

(3) Questions about the FoF-VRET treatment experience, including whether they underwent other FoF treatments \pm 1 year before/after the FoF-VRET treatments.

\section{Main outcome measures}

The primary measure of FoF-VRET efficacy was number of flights per month (FpM). The secondary outcome measure was number of flight hours per month (FHpM). For example, a participant interviewed 18 months after VRET completion reported the following flight information: to New York (11h) in month +2 , to London $(5.5 \mathrm{~h})$ in month +7 , and to Eilat $(1 \mathrm{~h})$ in month +17 . His/her outcome measures were thus $F p M=(3 / 18)$ and 
Efficacy of a VRET for FOF

$139 \mathrm{FHpM}=(17.5 / 18)$. Corresponding pre-treatment measures were calculated from data for

140 the identical period of pre-treatment time. An indirect measure of treatment efficacy was

141 reported anxiety level for pre- and post-treatment flights.

\section{FoF-VRET treatment}

Refer to the Supplementary Material for a brief description of the FoF-VRET treatment (for a full description see Czerniak, E., Caspi, A., Litvin, M., Amiaz, R., Bahat, Y., Baransi, H., . . Plotnik, M. (2016). A novel treatment of fear of flying using a large virtual reality system. Aerospace medicine and human performance, 87(4), 411-416.).

\section{Statistical analyses}

Non-parametric within-participant analyses (Wilcoxon signed-rank tests) were used to compare pre- and post-treatment FpM, FHpM and anxiety levels. Alpha level was set at $p<.05$, two-tailed.

\section{Flight activity before and after FoF-VRET treatment}

\section{RESULTS}

$$
\text { Participants showed a clear increase in flight activity post-treatment as compared to }
$$

pre-treatment (Figure 1).

\section{Figure 1 goes here}

Regarding flight activity outcomes before and after treatment, within-participant analyses revealed a significant difference for FpM and FHpM before (FpM: median=0, Interquartile Range $(\mathrm{IQR})=.07, \mathrm{FHpM}$ : median=0, $\mathrm{IQR}=.28)$ and after $(\mathrm{FpM}$ : median=.13, IQR=.18, FHpM: median=.57, IQR=1.04) treatment (Wilcoxon signed-rank tests, $Z=-6.89, p<.0001, Z=-6.73, p<.0001$, respectively). 
Efficacy of a VRET for FOF

161

162

163

164

165

166

167

168

169

170

171

172

173

174

175

176

177

178

179

180

181

182

183

Figure 2 shows FpM and FHpM across participants in the months before and after treatment.

Figure 2 goes here

Anxiety before and after FoF-VRET

Within-participant analyses (Wilcoxon test) revealed a significant difference in selfreported anxiety level for flights before (median=6.0, IQR=2.0) and after (median=4.0, $\mathrm{IQR}=2.2$ ) the treatment (Wilcoxon signed-rank test, $Z=-5.16, p<.0001$ ). Figure 3 shows anxiety level for flights before and after FoF-VRET.

Figure 3 goes here

Reasons for seeking FoF-VRET and other FoF treatments

To provide additional clinical background, Figure 4 shows the distribution of reasons for seeking FoF-VRET across participants, as reported during the phone interview.

Figure 4 goes here

Figure 5 shows the distribution of other treatments for FoF within approx. \pm 1 year of FoF-VRET reported by participants. The distribution indicates that nearly half $(48 \%)$ of participants did not engage in any other treatment. Among the other participants, psychological treatment (18\%) and FoF workshops (12\%) were most common; hypnosis (4\%) and CBT (3\%) were least common.

Figure 5 goes here

To confirm that our findings regarding FoF-VRET efficacy were not unduly affected by the additional treatments, we conducted a post hoc analysis. Briefly, we split participants into 'no other treatment' (48\%), and 'other treatment' groups. For each participant, we computed pre/post change (post-treatment minus pre-treatment; $\Delta$ ) in 
Efficacy of a VRET for FOF

184 FpM and FHpM, respectively. Man-Whitney tests revealed no significant differences

185 between the groups ( $\triangle \mathrm{FpM}: U=1397.5, p=.56, \Delta \mathrm{FHpM}: U=1379.5, p=.64)$, suggesting

186 that other treatments did not appreciably affect the increased flight activity we attribute to

187 FoF-VRET.

This study aimed to determine the efficacy of FoF-VRET treatment using a 190 retrospective follow-up questionnaire conducted over the phone. Our study is novel in 191 that we evaluated individuals who voluntarily paid for and received treatment in our 192 virtual reality center.

This FoF-VRET has several advantages over standard FoF exposure treatments:

194 Firstly, it provides a safe, controlled environment that can be continuously monitored and manipulated by professional therapists and technicians. Secondly, it provides a highly

196 detailed visual, auditory, and motion simulation of an actual flight experience rather than

197 a static airplane. This provides better exposure to the fear-triggering factors, potentially

198 inducing participant responses more similar to those elicited by real air travel. Finally,

199 other measures like heart rate and blood pressure can be recorded during VR exposure

200 therapy to provide therapists with more comprehensive clinical information. Efficacy

203 significantly increased, reflecting treatment efficacy. Additionally, in-flight anxiety level 204 significantly declined following treatment. These results are in line with other 205 retrospective follow-up studies assessing the efficacy of other virtual reality-based FoF 206 treatments $(17,18,20,21,24,25)$. The results of this study corroborate these prior 
Efficacy of a VRET for FOF

207 studies and provide new evidence that those who benefited from the treatment continue to

208 fly as long as eighteen months after FoF-VRET treatment initiation.

209 While previous studies evaluating the efficacy of FoF-VERT used air travel in the

210 post-treatment period (i.e., yes/no) as the sole (binary) outcome (e.g. $(24,25)$ ), the current

211 study introduces additional measures: flight frequency (i.e., number of flights per month)

212 and flight hours per month in the post-treatment period. We believe that with the addition

213 of these measures, we are able to provide better evidence of treatment efficacy, as we

214 show that treated participants no only fly more often, but also that they fly for longer

215 durations. These results suggest that engaging in FoF-VRET leads participants to take

216 flights they would not have been prepared to take prior to treatment.

217 In a recent meta-analysis of 11 randomized controlled trials, Cardos et al. (23)

218 reported significant overall efficacy of a FoF-VRET intervention $(G=0.592)$ and a

219 significant increase in flight activity at follow-up $(G=0.588)$, demonstrating the

220 advantage of FoF-VRET treatment over control/traditional FoF treatments. However,

221 their results also reveal the limitations of these trials due to poor study quality and small

222 sample size. The authors suggest that reported effects may have been overestimated as a

223 result of these issues. In contrast, our findings are based on a larger sample size and a

224 more true-to-life (ecological) environment than those of the aforementioned studies.

Other results

226 Some of our results elucidate clinical aspects of FoF and its treatment. While most 227 participants reported suffering specifically from FoF (acrophobia), a significant number 228 of participants reported suffering from general anxiety. Furthermore, almost half (48\%) 229 of the individuals receiving FoF-VRET treatment reported that they did not engage in any 
Efficacy of a VRET for FOF

230 other treatments at least one year prior to treatment, suggesting that half of those

231 suffering from FoF are untreated and may avoid air travel.

232

\section{List of abbreviations}

\section{Conclusions}

\section{Limitations and future work}

The current study is limited in several important ways. Firstly, to maximize sample size, we did not collect data at a fixed length of time from treatment (e.g., one year). Consequently, some adjustments to the data were required (e.g., standardizing the primary outcome measures to permit within-subject statistical comparisons). Secondly, the attrition rate was relatively high $(51.8 \%)$, which may have affected the results. Although this level of attrition was higher than in other retrospective follow-up studies (13\% in Rothbaum et al. (24), $29.3 \%$ in Tortilla-Feliu et al. (18), 10\% in Wiederhold et al. (25) and $10 \%$ in Muhlberger et al. (17)), a higher attrition rate may be expected for participants solicited to participate in a phone survey following receipt of a clinical treatment they paid for as compared to participants volunteering in research studies.

Current results are indicative of FoF-VRET treatment efficacy. Air travel is an integral part of modern life in the industrialized world, and its prevalence is expected to grow as airfares continue to decrease and global economics entails more business travel (1). We can therefore expect a heightened awareness of FoF and an increase in referrals for suitable treatments including VRETs. Future studies should evaluate long-term maintenance of the treatment effect and consequently identify the ideal frequency for delivery of subsequent booster treatments. 
Efficacy of a VRET for FOF

253 CATR - Center of Advanced Technologies in Rehabilitation; CBT - Cognitive

254 Behavioral Therapy; FHpM - Flight Hours per Month; FoF - Fear of Flying; Fpm -

255 Flight per Month; IQR - Interquartile Range; VR - Virtual Reality; VRET - Virtual

256 Reality Exposure Treatment

257 Acknowledgments: We would like to thank Ms. Yarden David and Ms. Yael Menkes,

258 for performing some of the phone surveys.

259 


\section{REFERENCES}

261

262

263

264

265

266

267

268

269

270

271

272

273

274

275

276

277

278

279

280

281

282

283

284

285

286

287

288

289

290

291

292

293

294

295

296

297

298

299

300

301

302

303

304

305

306

307

308

309

310

311

312

1. (IATA) TIATA. IATA's annual
https://www.iata.org/publications/Pages/annual-review.aspx.

2. Transportation USDo. Transportation Fatalities by Mode 2017 [Available from: https://www.bts.gov/content/transportation-fatalities-mode.

3. Dean RD, Whitaker KM. Fear of flying: Impact on the US air travel industry. Journal of Travel Research. 1982;21(1):7-17.

4. Oakes M, Bor R. The psychology of fear of flying (part I): A critical evaluation of current perspectives on the nature, prevalence and etiology of fear of flying. Travel medicine and infectious disease. 2010;8(6):327-38.

5. Van LG, Diekstra R. Fear of flying treatment programs for passengers: an international review. Aviation, space, and environmental medicine. 2000;71(4):430-7.

6. Ferrand M, Ruffault A, Tytelman X, Flahault C, Négovanska V. A cognitive and virtual reality treatment program for the fear of flying. Aerospace medicine and human performance. 2015;86(8):723-7.

7. Czerniak E, Caspi A, Litvin M, Amiaz R, Bahat Y, Baransi H, et al. A novel treatment of fear of flying using a large virtual reality system. Aerospace medicine and human performance. 2016;87(4):411-6.

8. Kraaij V, Garnefski N, Van Gerwen L. Cognitive coping and anxiety symptoms among people who seek help for fear of flying. Aviation, space, and environmental medicine. 2003;74(3):273-7.

9. Foreman EI, Bor R, van Gerwen L. The nature, characteristics, impact and personal implications of fear of flying. Aviation Mental Health: Psychological implications for Air Transportation. 2006:53-68.

10. Greist JH, Greist GL. Fearless flying: A passenger guide to modern airline travel: Taylor Trade Publications; 1981.

11. Wiederhold BK, Gevirtz RN, Spira JL. Virtual reality exposure therapy vs. imagery desensitization therapy in the treatment of flying phobia. Towards cyberpsychology: Mind, cognition and society in the internet age. 2001:253-72.

12. da Costa RT, Sardinha A, Nardi AE. Virtual reality exposure in the treatment of fear of flying. Aviation, space, and environmental medicine. 2008;79(9):899-903.

13. Rothbaum BO, Anderson P, Zimand E, Hodges L, Lang D, Wilson J. Virtual reality exposure therapy and standard (in vivo) exposure therapy in the treatment of fear of flying. Behavior therapy. 2006;37(1):80-90.

14. Rus-Calafell M, Gutiérrez-Maldonado J, Botella C, Baños RM. Virtual reality exposure and imaginal exposure in the treatment of fear of flying: A pilot study. Behavior modification. 2013;37(4):56890.

15. Botella C, Serrano B, Baños RM, Garcia-Palacios A. Virtual reality exposure-based therapy for the treatment of post-traumatic stress disorder: a review of its efficacy, the adequacy of the treatment protocol, and its acceptability. Neuropsychiatric disease and treatment. 2015;11:2533.

16. Schultheis MT, Rizzo AA. The application of virtual reality technology in rehabilitation. Rehabilitation psychology. 2001;46(3):296.

17. Muhlberger A, Wiedemann G, Pauli P. Efficacy of a one-session virtual reality exposure treatment for fear of flying. Psychotherapy Research. 2003;13(3):323-36.

18. Tortella-Feliu M, Botella C, Llabrés J, Bretón-López JM, del Amo AR, Baños RM, et al. Virtual reality versus computer-aided exposure treatments for fear of flying. Behavior Modification. 2011;35(1):330.

19. Krijn M, Emmelkamp PM, Ólafsson RP, Bouwman M, Van Gerwen LJ, Spinhoven P, et al. Fear of flying treatment methods: virtual reality exposure vs. cognitive behavioral therapy. Aviation, space, and environmental medicine. 2007;78(2):121-8.

20. Maltby N, Kirsch I, Mayers M, Allen GJ. Virtual reality exposure therapy for the treatment of fear of flying: A controlled investigation. Journal of Consulting and Clinical Psychology. 2002;70(5):1112.

21. Rothbaum BO, Hodges L, Smith S, Lee JH, Price L. A controlled study of virtual reality exposure therapy for the fear of flying. Journal of consulting and Clinical Psychology. 2000;68(6):1020.

22. Wiederhold BK, Jang DP, Gevirtz RG, Kim SI, Kim I-Y, Wiederhold MD. The treatment of fear of flying: a controlled study of imaginal and virtual reality graded exposure therapy. IEEE transactions on information technology in biomedicine. 2002;6(3):218-23. 
Efficacy of a VRET for FOF

23. Cardoş RA, David OA, David DO. Virtual reality exposure therapy in flight anxiety: a quantitative meta-analysis. Computers in Human Behavior. 2017;72:371-80.

24. Rothbaum BO, Hodges L, Anderson PL, Price L, Smith S. Twelve-month follow-up of virtual reality and standard exposure therapies for the fear of flying. Journal of consulting and clinical psychology. 2002;70(2):428.

25. Wiederhold BK, Wiederhold MD. Three-year follow-up for virtual reality exposure for fear of flying. Cyberpsychology \& Behavior. 2003;6(4):441-5.

\section{Figure Legends}

Figure 1. Flight activity 18 months before and after FoF-VRET treatment for individual participants. A. Each point represents at least one flight for the given month (see key below panel). Negative values on the abscissa reflect months pre-treatment, and positive values reflect months post-treatment, vertical orange line represents the month during which the treatment took place. Each horizontal row represent data from one participant. Data from 17 participants who did not fly before or after the treatment (i.e, reciprocal 18 months periods pre- and post-treatment) are not shown, yet these data were included in statistical analyses. Following treatment, mean \pm SD FpM increased from $.05 \pm .07$ to $.16 \pm .07$ flights ( $\mathrm{n}=103$; see also text for non-parametric comparisons). B. Mean flights hours per month (FHpM) across participants. Following treatment, mean FHpM rose from $.22 \pm .41$ to $.80 \pm .86$ hours per month. Note that for each participant, pre-treatment data were analyzed for the identical length of time as the post-treatment period at the time of data collection (see text). Thus, for all 103 participants, data was analyzed for 6 months pre/post treatment (red lines), for 64 participants data was analyzed for 12 months pre/post treatment (green lines), and for 35 participants data was analyzed for 18 months pre/post treatment (black lines). Pre-hoc analyses confirmed uniformity of distributions during overlapping periods for all three groups. 
Efficacy of a VRET for FOF

341 Figure 2. Mean flights per month (FpM; left) and flight hours per month (FHpM; right) 342 across participants. Negative values on the $x$-axis reflect months pre-treatment, and 343 positive values reflect months post-treatment. Following treatment, mean \pm SD FpM 344 increased from $.05 \pm .07$ to $.16 \pm .07$ flights; mean FHpM rose from $.22 \pm .41$ to $.80 \pm .86$

345 hours per month. Note that for each participant, pre-treatment data was analyzed for the 346 identical length of time as the post-treatment period at the time of data collection (see 347 text). Thus for all 103 participants, data was analyzed for 6 months pre/post treatment 348 (red lines), for 64 participants data was analyzed for 12 months pre/post treatment (green 349 lines), and for 35 participants data was analyzed for 18 months pre/post treatment (black 350 lines).

351 Figure 3. Level of self-reported anxiety across participants for flights before and after 352 FoF-VRET (box plots). The scale ranged from 1 (least anxious) to 7 (most anxious).

353 Figure 4. Distribution of reasons for seeking FoF-VRET treatment across participants. 354 Note: For participants who reported more than one reason, all reported reasons are 355 included.

356 Figure 5. Distribution of other FoF treatments within one year of FoF-VRET. Note: For 357 participants who reported more than one treatment, all reported treatments are included. 


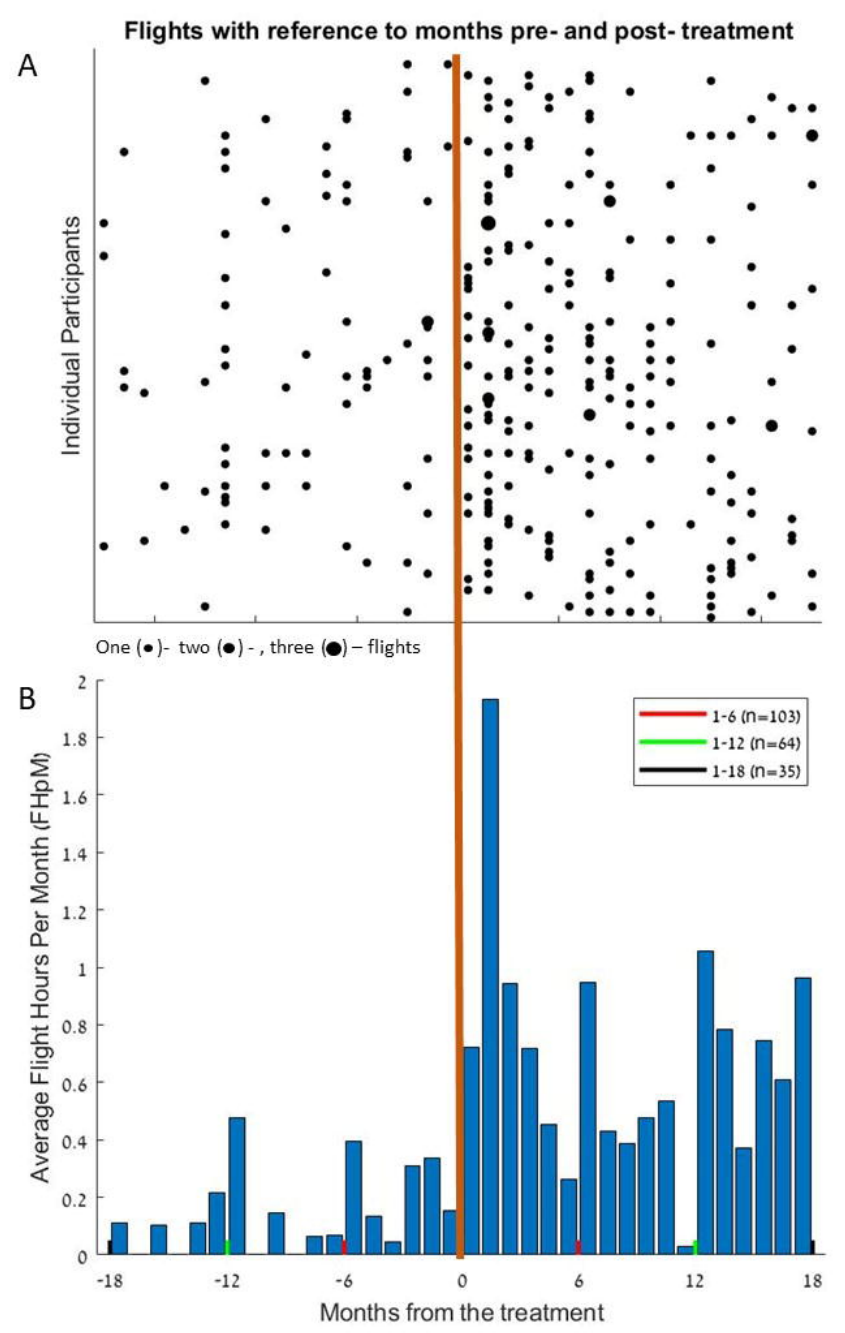




\section{FpM}

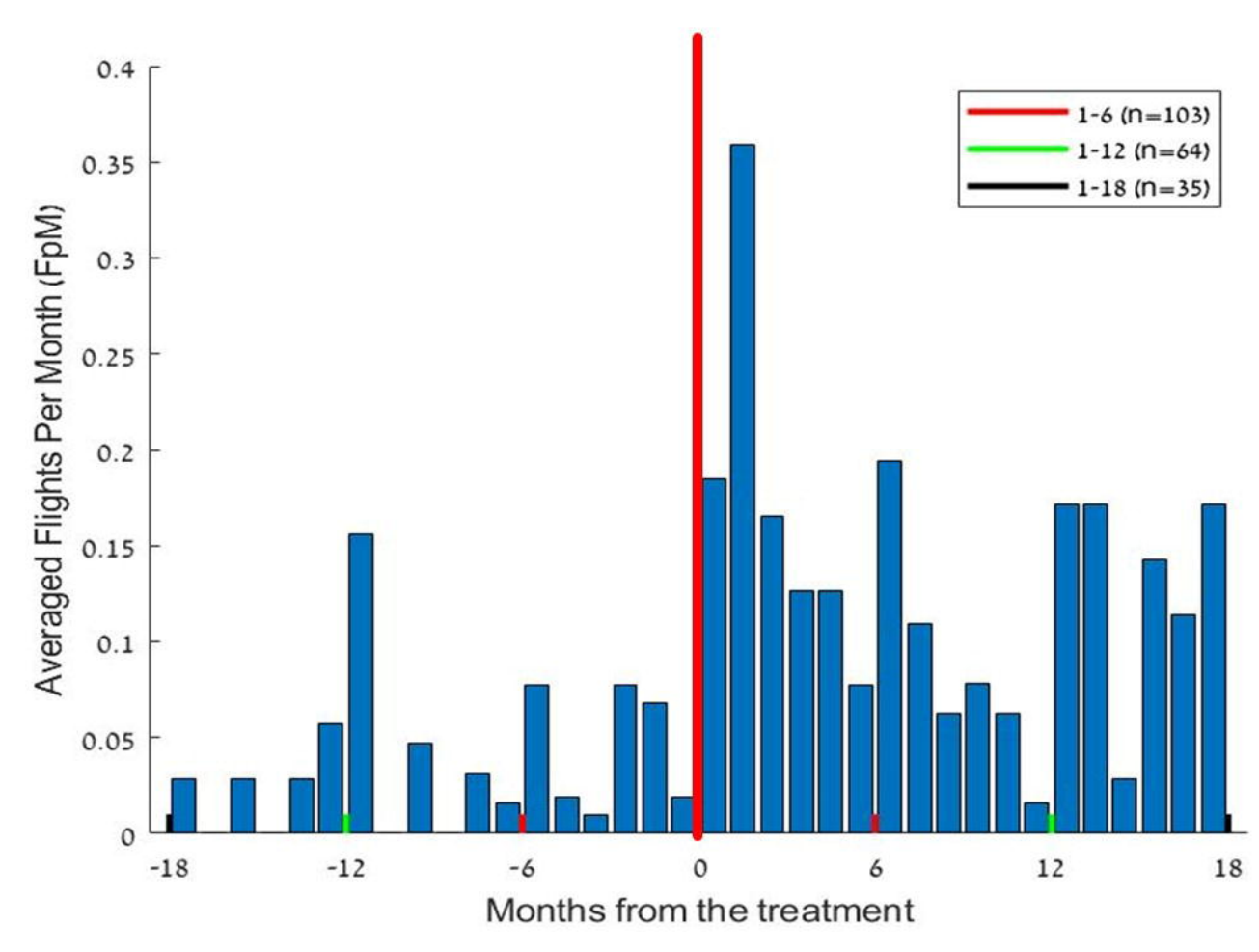

\section{FHpM}

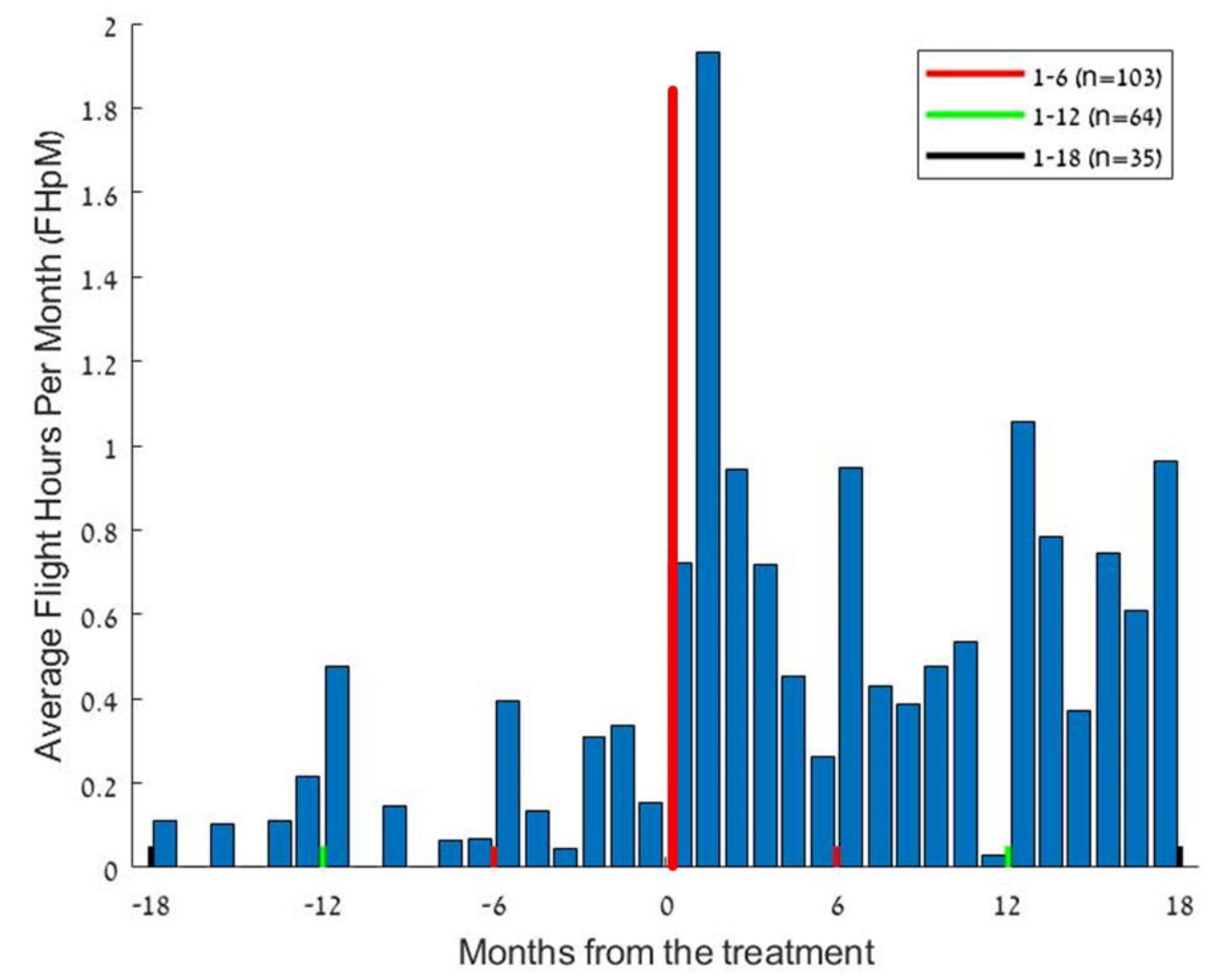




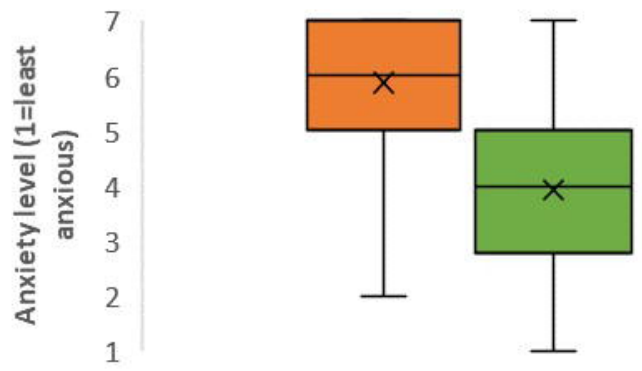

$\square$ Before FoF-VRET $\square$ After FoF-VRET 


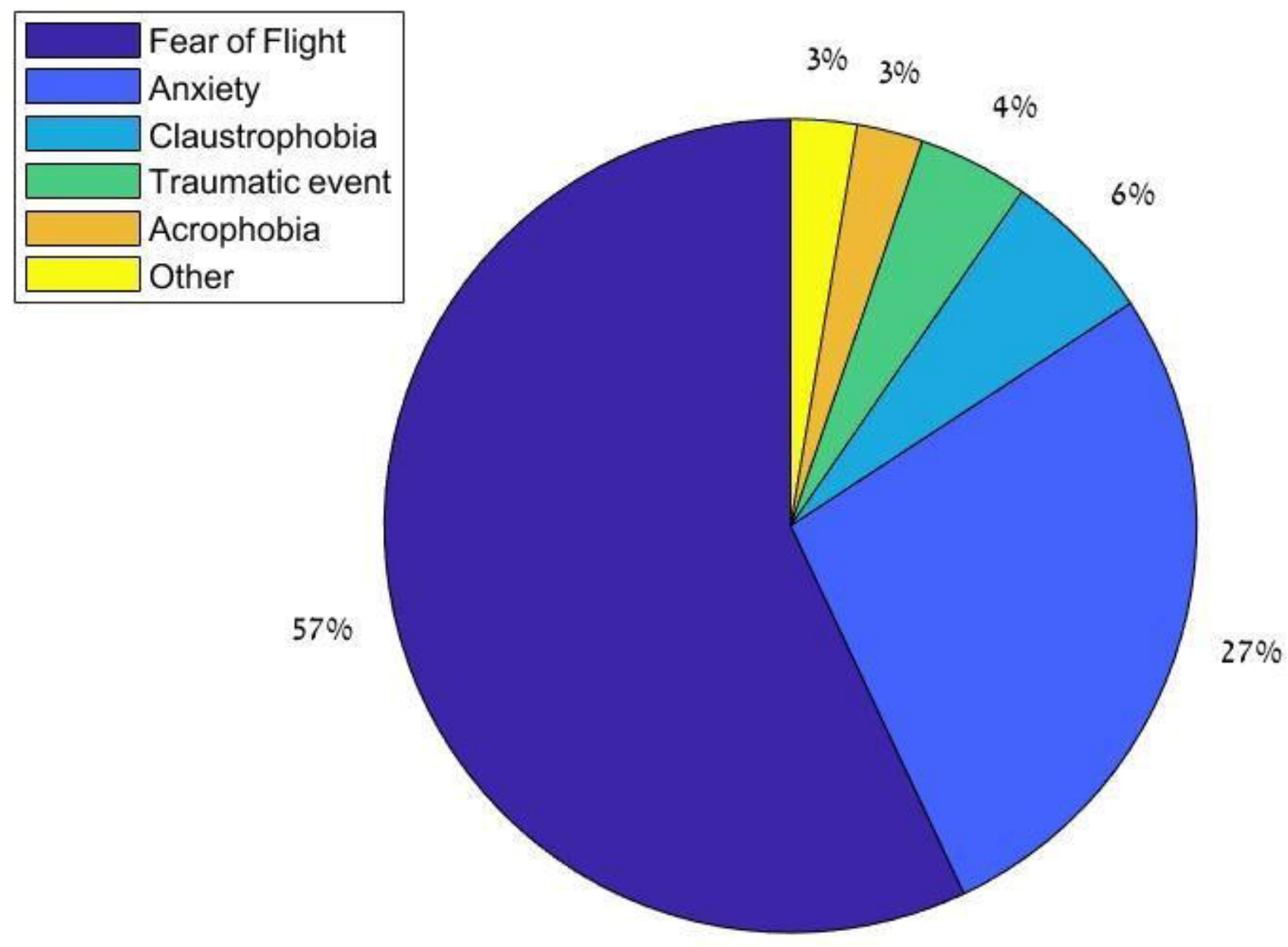




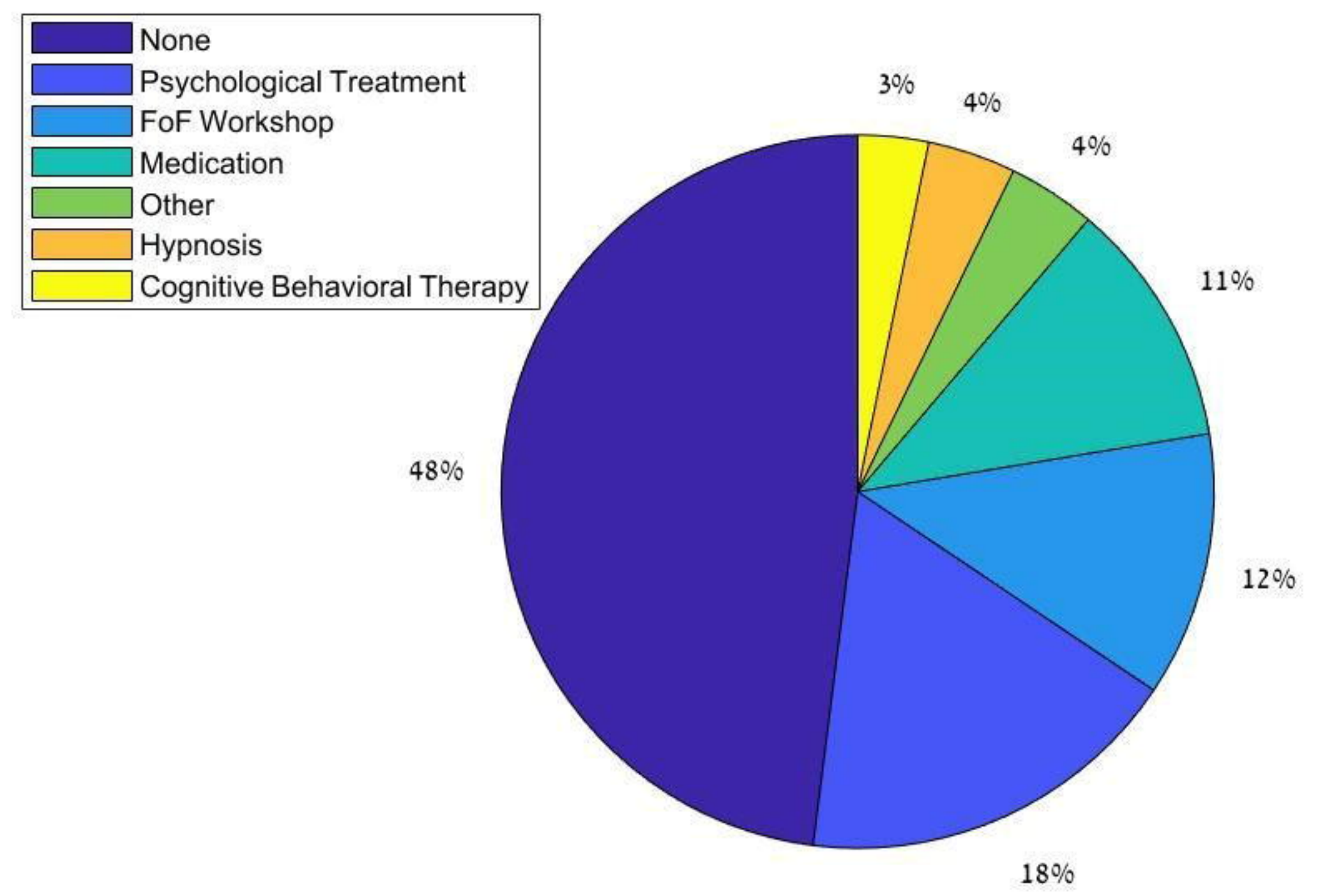

